\title{
Patterns of physical activity, adherence to a wearable activity tracker during a 12-week intervention and correlation between physical activity and function in individuals with hip and/or knee osteoarthritis
}

Elin Östlind ( $\nabla$ elin.ostlind@med.lu.se)

Lunds universitet Medicinska fakulteten https://orcid.org/0000-0001-9725-1786

\section{Anita Sant'Anna}

Viniam Consulting $A B$

\section{Frida Eek}

Lunds universitet Medicinska fakulteten

\section{Kjerstin Stigmar}

Lunds universitet Medicinska fakulteten

\section{Eva Ekvall Hansson}

Lunds universitet Medicinska fakulteten

\section{Research article}

Keywords: Hip osteoarthritis, knee osteoarthritis, physical activity, wearable activity tracker, mhealth, Fitbit

Posted Date: September 18th, 2020

DOI: https://doi.org/10.21203/rs.3.rs-70745/v1

License: (c) (1) This work is licensed under a Creative Commons Attribution 4.0 International License. Read Full License 


\section{Abstract}

\section{Background}

Osteoarthritis is a common joint disorder causing pain, functional limitations and an increased risk of comorbidities. Physical activity (PA) is the standard treatment but the majority of individuals with OA do not meet the recommended levels of PA (150 min/week). Monitoring PA with a wearable activity tracker (WAT) could promote PA but increased knowledge on PA patterns and usage of a WAT is needed. The aim of this study was to describe PA patterns and adherence to using a WAT among participants of working age with hip and/or knee $\mathrm{OA}$, during a 12-week intervention. In addition, this study explores the correlation between baseline joint function and subsequent PA.

Methods

Individuals of working age with hip and/or knee OA $(n=75)$ who used a WAT, Fitbit Flex 2, for 12 weeks as part of an intervention in a cluster-randomized controlled trial were included. Participants were encouraged to monitor their PA in the Fitbit-app. An activity goal of 7,000 steps per day was set. Activity data was collected from the Fitbit. HOOS/KOOS were completed at baseline.

Results

Participants walked on average 10593 (SD 3431) steps per day, spent 336.0 (SD 249.9) minutes in moderate/vigorous (MV) PA and used the Fitbit for $88.4 \%$ of the 12 -week intervention period. Steps per day decreased significantly over the 12 weeks ( $\beta$-coefficient $-117,95 \% \mathrm{Cl}-166$ to $-68, p=<0.001$ ) as well as MVPA per week ( $\beta$-coefficient $-3.5,95 \% \mathrm{Cl}-6.5$ to $-0.5, p=0.02$ ) and adherence to using the Fitbit ( $\beta$ coefficient $-1.3,95 \% \mathrm{Cl}-1.8$ to $-0.8, \mathrm{p}=<0.001)$. There were no significant correlations between baseline function (HOOS/KOOS) and subsequent PA.

\section{Conclusion}

The majority of participants reached 7,000 steps/day and the recommended 150 minutes in MVPA per week. However, weekly PA decreased gradually over time. Adherence to using the Fitbit was high but also decreased during the intervention. Understanding PA-patterns and the use of a Fitbit to promote PA could be beneficial in tailoring interventions for individuals with hip and/or knee OA.

Trial registration

The C-RCT was registered in ClinicalTrials.gov. Registration number: NCT03354091. Registered $11 / 27 / 2017$.

\section{Background}


Osteoarthritis (OA) is one of the most common musculoskeletal disorders in the world (1), causing pain (2), disability and reduced quality of life (3). OA can affect any synovial joint, but the knee, hip and hand are the most common sites (4). Knee OA is more common in women and the prevalence levels out at 50 years of age while hip OA continues to increase with age (5). The prevalence of physician-diagnosed hip and knee OA was 5.8 and 13.8 respectively in a large population $\geq 45$ years in Sweden (4). OA represents a substantial burden for the individual as well as society due to increased work absenteeism and healthcare costs (3). Many individuals with OA have one or several comorbidities such as hypertension, dyslipidemia and diabetes (6). Studies have also shown an increased risk of cardiovascular disease (7), and death due to cardiovascular disease in individuals with OA compared to individuals without OA (8).

The recommended first-line treatment of OA is physical activity (PA); it reduces pain, improves physical function and health-related quality of life for individuals with lower limb OA (9). The World Health Organization (WHO) defines PA as "any bodily movement that requires energy expenditure" while exercise is defined as "a subcategory of PA that is planned, structured, repetitive and aims to improve or maintain one or more components of physical fitness." Exercise is superior to all other non-operative treatments for meaningful improvements of OA pain (10). The type of exercise does not seem to matter. Previous research has shown that aquatic or land-based exercise as well as strength training, aerobic exercise or tai chi were associated with reduction in pain and improvement in physical function and health-related quality of life (9). Moderate or vigorous PA (MVPA) for at least 150 min per week is recommended to all adults by the WHO to reduce the risk of all-cause mortality, coronary heart disease, type 2 diabetes, depression and several other diseases (11). These recommendations have also been converted to steps per day and correspond to approximately 7,000-11,000 steps per day (12). However, a majority of individuals with hip and/or knee OA do not meet these PA recommendations $(13,14)$.

One reason for persons not meeting PA recommendations could be that individuals with OA often experience pain and reduced function in affected joints (15), which has been associated with level of PA $(16,17)$. Previous studies have reported that low physical function is related to low level of physical activity in individuals with hip and knee OA (18) and that higher levels of PA are associated with higher self-reported and objective functional measures in older adults (19). Veenhof et al. (18) concluded that individuals with low physical function need additional stimulation from clinicians to be more physically active. Identifying individuals with low physical function may therefore be important to predict level of PA.

Treatment strategies that include self-management skills to support the maintenance of PA, rather than supervised exercise, may be cost-effective and important in the treatment of OA $(20,21)$. Behavioral change techniques (BCTs) can improve self-management skills and are used in interventions to increase adherence to exercise. Patient led goal-setting, self-monitoring of behavior and non-specific rewards are examples of BCTs that are effective in improving adherence to PA in both short and long-term (22). A relatively novel and popular method that applies BCTs and has shown to increase PA in healthy populations as well as in populations with $\mathrm{OA}$ and other chronic conditions is the utilization of wearable 
activity trackers (WATs) (23-25). WATs are sensors that track movement and can be paired with a smartphone, tablet or computer application (App). A WAT can be worn on different locations, most commonly on the wrist, and are manufactured by large companies such as Fitbit, Jawbone, Apple, Polar and Nike. WATs have gained increased attention from the general population as well as researchers and clinicians during the last decade with a twenty-fold increase in number of WAT-studies, from 8 in 2013 to 199 in 2017 (26).

WATs have been suggested as a complement to traditional short-term exercise interventions to increase the long term adherence to PA-participation (23). The concept adherence has been defined by the WHO as "the extent to which a person's behavior - taking medication, following a diet, and/or executing lifestyle changes, corresponds with agreed recommendations from a healthcare provider" (27). For home exercises, a high adherence is associated with better outcomes on pain, physical function and selfperceived effect in patients with hip or knee OA but the adherence to exercise decreases over time (28). Adherence to using a WAT in a PA-intervention has been analyzed by a few studies in different populations $(25,29-33)$. Different definitions of adherence were used in these studies but overall, they showed a high short-term adherence to using the WAT. To our knowledge, there are no studies exploring adherence to WAT use in individuals of working age with OA using a Fitbit Flex 2.

The aim of this study was to describe patterns of PA and adherence to using a WAT among individuals of working age with hip and/or knee OA, during a 12-week intervention. A secondary aim was to explore the correlation between baseline self-reported function and subsequent PA.

\section{Methods}

\section{Design}

In this exploratory study, we conducted an analysis of WAT-data collected during a cluster-randomized trial (C-RCT). The C-RCT is registered in clinical trials (No: NCT03354091) (34). The underlying C-RCT compared treatment as usual (participating in a Supported Osteoarthritis Self-management Program) with treatment as usual together with self-monitoring PA using a commercial WAT, Fitbit Flex 2, for 12 consecutive weeks as an add-on. This study reports the PA data of the intervention group only.

\section{Setting}

Participants in this study took part of the Supported Osteoarthritis Self-management Program (SOASP). The SOASP is recommended in clinical guidelines for the management of patients with OA in the hip, knee and hand in Sweden $(35,36)$. The SOASP offers first line treatment for patients with hip, knee and/or hand OA and is generally offered in primary health care. The minimal intervention in the SOASP is two theoretical group sessions including information about $\mathrm{OA}$, exercise and self-management held by a physiotherapist (PT). After the theoretical sessions, patients are offered an individual appointment with a PT and are introduced to specific exercises based on the patient's needs and goals. Some healthcare 
centers or physiotherapy clinics offer supervised group training, often for a limited period, e.g. two times a week for six weeks.

\section{Participants}

In this study, we included participants from the intervention group in the C-RCT. Eligible for recruitment in the C-RCT were individuals of working age in southern Sweden with hip and/or knee OA. The inclusion criteria were: working $\geq 50 \%$ (20 hrs. /week), aged between 18-67 years, being able to understand Swedish in speech and writing and able to participate in PA. They also had to have access to a smartphone, tablet or computer to use the Fitbit-app and be able to wear a WAT for 12 weeks. In this study, we included all patients that were randomized to the intervention group $(n=86)$. Six participants dropped out before the intervention, three participants dropped out due to technical problems and two due to low registration ( $<50 \%$ of the time period). In all 75 participants were included in the final analyses.

Potential participants in this study were approached in two different ways. PTs at healthcare centers and physiotherapy clinics in southern Sweden were contacted in 2017-2018 and asked to inform individuals participating in the SOASP about the research project. Participants in the SOASPs were given oral and written information about the research project, inclusion/exclusion criteria and how to register. They received this information from EÖ or the PT that held the theoretical sessions. The individuals selfregistered on the project's website using an electronic identification (ID) service (37) and thereby gave their informed consent. After a year of recruiting individuals, a Facebook advertisement was added to recruit additional participants in 2018-2019. The advertisement reached individuals between 4064 years of age living within a defined geographical area in southern Sweden. Individuals $(n=267)$ that were interested e-mailed EÖ and then received more information about the research project, inclusion/exclusion criteria and how to register. Sixty individuals registered on the project's website and took part of a SOASP offered within the research project. EÖ was responsible for the SOASPs held within this project $(n=9)$ that consisted of three theoretical sessions. An individual visit with a PT (EÖ) was also offered. Each SOASP was cluster-randomized using sealed envelopes exactly as the other SOASPs.

Intervention

The intervention in the C-RCT was the addition of PA self-monitoring with a wrist-worn WAT, the Fitbit Flex 2. In connection to participating in the SOASP, each participant met with EÖ and received the Fitbit. They were aided in installing the Fitbit application (app) and synchronizing the device to the participant's app as well as connecting the participant's FitBit account to the study via the project's website. The participants in the intervention group were asked to wear the Fitbit for 12 weeks, from morning until bedtime. They were also asked to monitor their activity by using the app once a day. Using the app once per day allowed for synchronization of the data from the device to the app. During the intervention, the participants were only contacted by EÖ if there were a great number of days without registered activity. The default activity goal of 10,000 steps per day was changed to 7,000 because we wanted it to be achievable for the participants. Previous research has also suggested that 7,000 steps per day might be 
an accurate estimate for meeting the recommended 150 min per week of MVPA $(12,38)$. The other default activity goals in the app remained unchanged.

Outcomes and measurements

The outcomes in this study were level of PA, and self-reported function. PA was monitored by Fitbit Flex 2, a commercial accelerometer-based WAT that continually estimates steps taken, distance traveled, and time in different activity levels. The Fitbit is waterproof and can be worn during swimming and showering. The device is worn inside a rubber wristband and has five small LED-lights but no display. The measurements are transmitted via Bluetooth from the device to a smartphone, tablet or computer app, which in turn transfers the data to the Fitbit servers. All registered activity data can be viewed anytime on the app or on the Fitbit user portal.

In this study, we considered only the number of steps and the time spent in different activity levels as estimated by the Fitbit. Fitbit uses a proprietary algorithm to estimate metabolic equivalents (METs). One MET is described as the energy expended during rest or sitting quietly. An activity is considered moderately or vigorously active if it continues for $>10$ minutes and exceeds 3 respectively 6 METs (39, 40). The measured activity data is made available to third parties via Fitbits Web APIs (41). We developed our own data server, which would query the Fitbit's Web API every evening for activity from our study participants during the previous day. In particular, we requested number of steps, number of sedentary minutes, number of minutes in light, moderate, vigorous activity and total distance walked for every minute of the previous day. These variables are named in the Fitbit API respectively as "steps", "sedentaryMinutes", "lightlyActiveMinutes", "fairlyActiveMinutes", "veryActiveMinutes" and "total distance". In order to be able to query the Fitbit Web APIs we submitted a request to Fitbit and obtained approval for our research study. We requested that participants grant us access to their Fitbit accounts via our project website when they received the Fitbit from the researcher who monitored the study (EÖ).

Two systematic reviews on validity and reliability of commercial WATs has shown an overall high validity for steps and, to a lesser extent, duration of PA $(42,43)$. The reliability and validity of the Fitbit Flex and Fitbit Flex 2 have been evaluated by a few studies but the results are inconsistent. A majority of the studies reported that Fitbit Flex overestimated number of steps and time in MVPA compared to actigraph, which is commonly used as a 'gold standard' for measuring PA in free-living setting (44-46).

Subjective function was measured by the Hip injury and Osteoarthritis Outcome Score (HOOS) and Knee injury and Osteoarthritis Outcome Score (KOOS) $(47,48)$. HOOS/KOOS contain five subscales: Pain, Symptoms, Activities of Daily Living (ADL), Sport and Recreation function (Sport/Rec) and hip/kneerelated Quality of Life (QoL). Each subscale is scored separately from zero (extreme knee problems) to 100 (no knee problems). HOOS/KOOS have been shown to have adequate psychometric properties for individuals with OA (47-50). In this study, participants filled out HOOS or KOOS at baseline depending on their most affected joint.

\section{Data analysis}


The raw activity data from the Fitbit was pre-processed using Rstudio (51). The data from each participants was identified with an anonymous random string. We included data from days 2-85 in the analysis. Day 1 was excluded because, in most cases, participants only started using the Fitbit in the afternoon. All days after day 85 were excluded because we wanted to analyze twelve full weeks (84 days). For each participant, the number of minutes in MVPA on the same calendar day were summed together to reflect total number of "Active Minutes" that day. For each participant, any days during the intervention period for which we did not have any data were considered as "missing days" and excluded from subsequent analysis. Some days only had few steps and to avoid including them in the analysis, days with less than 1,500 registered steps were also excluded. This threshold has been used in previous research $(52,53)$.

The subsequent statistical analysis was undertaken in IBM SPSS Statistics for Windows, version 25 (IBM Corp., Armonk, N.Y., USA). If activity data were missing for 1 to 3 days in a given week, the data was imputed as the mean value of the remaining days that week. If more than 4 days of data were missing, the whole week was excluded from the analysis. For each participant and for each week, we considered the mean and standard deviation of the number of steps per day; the total number of minutes (sum) in MVPA; and the adherence to Fitbit use as the number of days with data that week divided by 7. A total percentage of Fitbit-use during the total intervention period was also calculated: number of valid days/84.

To explore patterns of physical activity and adherence to using the Fitbit during the 12-week intervention, we carried out a linear mixed model with a random intercept to account for the weeks clustered within each participant. The dependence between repeated measurements was modelled using an autoregressive covariance structure $(A R(1))$. The linear trend ( $\beta$-coefficient) represents the total 12-week intervention and shows change per week. Correlations between baseline function for each $\mathrm{HOOS} / \mathrm{KOOS}$ subscale and mean number of steps per day and mean number of minutes in MVPA/week during the intervention were assessed by using Spearman's rank correlation.

\section{Results}

Participants

Seventy-five individuals (10 men, 65 women) with hip and/or knee OA completed the intervention and were included in this study. One individual did not fill out the questionnaire at baseline, characteristics are therefore presented for 74 individuals (Table 1).

Table 1. Participant characteristics and baseline data $(\mathrm{HOOS} / \mathrm{KOOS})(n=74)$ 


\begin{tabular}{|c|c|}
\hline Age (years), mean (SD) & $56.9(5.2)$ \\
\hline Gender (female), n (\%) & 64 (86.5) \\
\hline Married or living with partner, $\mathrm{n}(\%)$ & $56(75.7)$ \\
\hline \multicolumn{2}{|l|}{ Most affected joint, $n$ (\%) } \\
\hline Hip & 17 (22.7) \\
\hline Knee & $58(77.3)$ \\
\hline Education (postsecondary), n (\%) & $49(66.2)$ \\
\hline \multicolumn{2}{|c|}{ Employment (percentage of full time - $40 \mathrm{hrs}$.) $n(\%)$} \\
\hline $0-25 \%$ & $2(2.7)$ \\
\hline $26-50 \%$ & $5(6.8)$ \\
\hline $51-75 \%$ & $8(10.8)$ \\
\hline $76-100 \%$ & $58(78.4)$ \\
\hline Unemployed & $1(1.4)$ \\
\hline \multicolumn{2}{|l|}{ Physically demanding work, n (\%) } \\
\hline No & $52(70.3)$ \\
\hline Yes, several times a week & $7(9.5)$ \\
\hline Yes, daily & $14(18.9)$ \\
\hline Missing & $1(1.4)$ \\
\hline Sedentary work, sitting > 50\%, $n(\%)$ & $39(52.7)$ \\
\hline Missing & $2(2.7)$ \\
\hline \multicolumn{2}{|c|}{ Current self-assessed PA-level compared to PA-level before OA, $n(\%)$} \\
\hline More physically active & $8(10.8)$ \\
\hline Equally physically active & $41(55.4)$ \\
\hline Less physically active & $24(32.4)$ \\
\hline Missing & $1(1.4)$ \\
\hline \multicolumn{2}{|c|}{ Regular usage of a WAT during the last three months before the intervention, $n(\%)$} \\
\hline Yes & $29(39.2)$ \\
\hline No & $41(55.4)$ \\
\hline Missing & $4(5.4)$ \\
\hline
\end{tabular}




\begin{tabular}{|ll|}
\hline HOOS, mean (SD) & \\
\hline Symptoms & $46.8(18.2)$ \\
\hline ADL & $58,3(22)$ \\
\hline Sport/Rec & $63.1(22.7)$ \\
\hline QoL & $39.2(22.8)$ \\
\hline KOOS, mean (SD) & $40.6(18.4)$ \\
\hline Pain & \\
\hline Symptoms & $60.6(19.7)$ \\
\hline ADL & $52.2(20.4)$ \\
\hline Sport/Rec & $72(19.5)$ \\
\hline QoL & $27.5(25.5)$ \\
\hline HOOS Hip injury and Osteoarthritis Outcome Score & $43.8(19.1)$ \\
\hline KOOS Knee injury and Osteoarthritis Outcome Score & \\
\hline
\end{tabular}

Patterns of Fitbit-measured physical activity during the intervention

Participants walked on average 10593 (SD 3431) steps per day during the 12-week intervention and spent 48.1 (SD 35.5) minutes in MVPA per day. MVPA per week was on average 336.0 (SD 249.9) minutes. There was a slight gradual decrease in steps per day over the 12 weeks ( $\beta$-coefficient $-117,95 \%$ $\mathrm{Cl}-166$ to $-68, \mathrm{p}=<0.001$ ) (Fig. 1). Week 2 had the highest average number of steps per day with 11162 (SD 3830) steps, and week 11 the lowest number of steps with 9589 (SD 3169) steps per day. MVPA per week also decreased slightly over the 12 weeks ( $\beta$-coefficient $-3.5,95 \% \mathrm{Cl}-6.5$ to $-0.5, \mathrm{p}=0.02$ ) (Fig. 2). MVPA was highest in week 2 with 369.0 (SD 282.9) minutes per week and lowest in week 11 with 305.1 (SD 227.7) minutes. Sixty-five (86.7\%) participants had on average > 7000 steps per day, 35 (46.7\%) participants had on average $>10000$ steps per day and $58(77.3 \%)$ had on average $>150 \mathrm{~min}$ in MVPA per week.

Patterns of adherence to using the Fitbit during the intervention

The Fitbit was used on average $88.4 \%$ of the days during the 12-week intervention with highest adherence in week 2 (94.7\%) and lowest in week $12(80.5 \%)$. The adherence to using the Fitbit decreased gradually over the 12 weeks ( $\beta$-coefficient $-1.3,95 \% \mathrm{Cl}-1.8$ to $-0.8, \mathrm{p}=<0.001$ ) (Fig. 3).

Correlation between baseline function and subsequent PA 
No correlations were found between HOOS/KOOS subscales and subsequent PA (average number of steps/day and minutes in MVPA/week during the intervention) (Table 2).

Table 2. Correlation (Spearman's Rho) between HOOS/KOOS subscales and average number of steps per day, minutes in MVPA per week $(n=74)$.

\begin{tabular}{|lllll|}
\hline & Steps/day & p-value & MVPA/week & p-value \\
\hline Pain & 0.061 & 0.605 & 0.026 & 0.828 \\
\hline Symptoms & 0.027 & 0.818 & -0.003 & 0.978 \\
\hline ADL & 0.108 & 0.360 & 0.051 & 0.666 \\
\hline Sport/Rec & 0.195 & 0.103 & 0.097 & 0.422 \\
\hline QoL & 0.123 & 0.301 & 0.061 & 0.608 \\
\hline
\end{tabular}

\section{Discussion}

This study provides insights into Fitbit-measured PA patterns and adherence to using a Fitbit in individuals of working age with hip and/or knee OA during a 12-week intervention. The vast majority of the participants were highly active and reached on average the recommended minimum of $>150$ minutes in MVPA per week and the activity goal in the Fitbit-app > 7000 steps per day. The adherence to using the Fitbit was also high during the intervention. A significant but small decrease was seen in both PA and adherence to using the Fitbit during the intervention. There were no significant correlations between baseline function as measured with HOOS/KOOS and subsequent PA.

The results of this study show that the participants were highly active throughout the intervention as measured with the Fitbit. Even in the least active week, week 11, they walked on average almost 10,000 steps $(9,589)$ and had 305 minutes in bouted MVPA, twice the amount of the recommended minimum of 150 minutes per week (11). This study reports higher levels of PA compared to previous studies with objectively measured PA in participants with hip or knee $O A(14,38,54-56)$. A systematic review showed that participants with hip and knee $O A$ averaged 8,174 respectively 7,753 steps per day and 160 respectively $50 \mathrm{~min}$ of MVPA (in bouts of $\geq 10$ minutes) per week (38). A more recent study reported similar results with $17.2 \%$ men and $9.2 \%$ women meeting the recommended level of $\geq 150$ minutes a week of bouted MVPA (14) which is in contrast to this study with $77.3 \%$ of participants reaching $\geq 150$ minutes per week of bouted MVPA. However, these results can be difficult to interpret due to differences in measurement. The most common accelerometer used in these studies was the non-commercial accelerometer Actigraph that is usually worn on the hip $(14,38)$. A few studies have used a Fitbit to measure PA in OA-populations as an outcome measure. These studies reported markedly lower number of steps per day compared to our study (54-56). A possible explanation for this could be differences in participant characteristics. In our study, the participants were younger and had better joint function (as measured with HOOS/KOOS) than the participants in the previous studies using Fitbit. 
The analyses of the activity data in this study showed a trend of a significant but small weekly decrease of 117 steps (daily average)/week and 3.5 minutes in MVPA (per week). This small decrease in PA may not be clinically important and after 12 weeks intervention, participants were still highly active. However, the decrease could indicate that it is difficult to change and maintain behavior and that PA-interventions often prove to be most effective in the short-term (57). Hartman et al. (32) also analyzed the weekly PApattern in an intervention using a Fitbit. In their study, MVPA per week also differed significantly but there was a slight increase in MVPA during the 12-week intervention, which is in contrast to the results in our study. One explanation for this could be the differences in follow-ups and reminders. Participants in that study received several phone calls and e-mails during the intervention (32).

Adherence to using the Fitbit was on average high throughout the 12 weeks but decreased slightly, gradually. The participants were contacted if data were missing for several days in a row, which could indicate technical problems. In some cases, the contacted participants had forgotten to charge the Fitbit. To charge and wear the device is a prerequisite for the utilization of WATs to be an effective method to increase and optimize PA (58). The average adherence of $88 \%$ in this study is comparable with the adherence presented in a recent systematic review in which three studies using wrist-worn WATs for 12 weeks reported an average adherence of $93 \%(25,29,30,33)$. In two of the three studies, participants had several follow-ups, which might have affected the adherence to using the WATs $(30,33)$. In studies with longer use of the Fitbit ( 9 and 12 months) and no follow-ups, the adherence was lower and had a gradual decrease. In the 9-month study, the overall adherence was only $44.5 \%$ and the authors concluded that participants who stopped wearing the device did not return to compliance on their own. They also reported that participants with positive attitudes toward technology had higher adherence to using the Fitbit (31). In the 12-month study, $50 \%$ used their Fitbit after six months and after 300 days, only $12 \%$ still used it. The most common reasons for participants to stop using the Fitbit were technological failures and empty batteries (59). In our study, we did not have any scheduled reminders to use the Fitbit but the contact that was made with participants lacking data for several days could also be a reminder to use the Fitbit. If no contact had been taken with the participants, the adherence in this study might have been lower.

This study also explored the correlation between baseline function measured with HOOS/KOOS and the subsequent PA measured with the Fitbit. In a previous study, individuals with OA described that their OArelated pain affected their ability to take part in PA and that they in some cases avoided activities that led to increased pain (2). Rosemann et al. (16) found that two of the main factors associated with lower levels of self-reported PA in participants with hip or knee OA were dysfunction in the lower limb and pain. Our hypothesis was therefore that the result on HOOS/KOOS subscales would be associated with subsequent level of PA and that this would be clinically important. If a lower score is associated with lower subsequent PA, this could help clinicians to identify individuals with OA that are in need of extra support to maintain/increase PA-levels. Heiberg \& Figved (60) reported that individuals who preoperative scored lower on HOOS subscales Pain and Sport/Rec were at risk of being less physically active in the long-term after a total hip arthroplasty. However, the results in this study showed weak and nonsignificant correlations between the subscales on HOOS/KOOS and level of PA (61). A reason for this 
might be that the participants in this study were more active and had overall better function and less pain as measured with HOOS/KOOS compared to other OA-populations $(13,14,50,60,62)$. The activity data was also collected during a PA-intervention that might have increased their PA compared to their ordinary levels of PA.

This study has some limitations. Our recruitment strategy with two different ways of approaching eligible participants was not optimal. We suspect that there was a selection bias, especially concerning participants that were recruited from the Facebook-advertisement. Compared with a large cohort including individuals with OA in Sweden that had participated in the SOASP, there were some major differences in patient characteristics in our study. The participants in our study were younger (57 compared to 67 years) but in this study, we only recruited individuals of working age. Our study also had a higher percentage of female participants ( $87 \%$ compared to $69 \%$ ) and a much higher proportion of participants with post-secondary education (66\% compared to $29 \%$ ) (63). In addition, almost $40 \%$ of the participants already regularly used a WAT prior to registering to this study. Our findings correspond well to the findings of a previous study by Macridis et al. (64) which reported that using a WAT was associated with being female, below 60 years of age, having a post-secondary education and meeting physical activity guidelines. Using Facebook to recruit participants is a cost-effective and time saving method but leads to an over representation of young, white women (65). Hence, our results may not be directly generalizable to the general OA-population.

The other major limitation is the measurement properties of the WAT used in this study, the Fitbit Flex 2. The choice of WAT was determined in consideration of several aspects such as popularity, cost and feasibility to extract data from the manufacturers' server. All commercial WATs have their limitations. Generally, wrist-worn WATs are less accurate than hip-worn WATs in measuring PA but are more userfriendly and therefore lead to higher adherence to usage $(25,66)$. In studies examining the measurement properties of the Fitbit Flex 2 and its predecessor, Fitbit Flex, the results are inconsistent but previous studies report that the Fitbit Flex overestimates steps and MVPA in free-living conditions (44-46). Since Fitbit is a commercial WAT, it uses a proprietary algorithm that only allows researchers access to already processed data and not the actual raw accelerometer data. This limits the interpretations of the data since the thresholds of different activities are unknown. The findings in this study should be seen in light of these limitations, which affect the interpretation and the generalization of the results.

\section{Conclusion}

In this study, we found that individuals of working age with hip and/or knee OA were highly active during the 12-week use of a wearable activity tracker and had a high adherence to using a WAT but there was a slight and gradual decrease in both PA and adherence. Compared to previous studies, participants in our study had a higher level of PA. There were no correlation between baseline function as measured with HOOS/KOOS and subsequent PA. We believe that the findings in this study may provide useful information about PA-patterns and adherence during an intervention in the OA population. Understanding 
the patterns of PA and the use of a WAT to promote PA could be beneficial in tailoring interventions for individuals with hip and/or knee OA.

Future research should include a more heterogeneous population of individuals with OA, especially individuals that are physically inactive and do not use a WAT regularly. Longer-term studies are also needed.

\section{Abbreviations}

\begin{tabular}{ll} 
OA & Osteoarthritis \\
\hline PA & Physical activity \\
\hline WHO & World Health Organization \\
\hline MVPA & Moderate and vigorous physical activity \\
\hline BCT & Behavior change techniques \\
\hline WAT & Wearable activity tracker \\
\hline C-RCT & Cluster-randomized controlled trial \\
\hline SOASP & Supported osteoarthritis self-management program \\
\hline PT & Physiotherapist \\
\hline EÖ & Elin Östlind \\
\hline MET & Metabolic equivalent \\
\hline HOOS & Hip injury and Osteoarthritis Outcome Score \\
\hline KOOS & Knee injury and Osteoarthritis Outcome Score \\
\hline SD & Standard deviation
\end{tabular}

\section{Declarations}

\section{Ethics approval and consent to participate}

This study was approved by the Regional Ethical Review Board in Lund, Sweden in conjunction with the C-RCT (2017/596). All participants received written information about the study and provided their informed consent with an electronic identification service before registering.

\section{Consent for publication}

Not applicable 
The datasets used and analyzed during the current study are available from the corresponding author on reasonable request.

\section{Competing interests}

The authors declare that they have no competing interests.

\section{Funding}

This study received fundings from the Swedish Research Council. The funder did not take part in designing the study, collecting data, analyzing, interpreting the results or writing the manuscript.

\section{Authors' contributions}

All authors (EÖ, AS, FE, KS, EEH) contributed in conceptualizing and designing the study. EÖ recruited participants, managed the intervention, handled the Fitbits and sent out questionnaires. AS collected and analyzed data from the Fitbit. FE and AS provided advice concerning statistical measurements. All authors (EÖ, AS, FE, KS, EEH) contributed in analyzing and writing the manuscript and all authors read and approved the final manuscript.

\section{Acknowledgements}

The authors would like to acknowledge Susann Ullén, Statistician at Clinical Studies Sweden- Forum South, Skåne University Hospital, for aiding us in statistical queries. We would also like to thank the participants in this study.

\section{References}

1. Murray CJL, Vos T, Lozano R, Naghavi M, Flaxman AD, Michaud C, et al. Disability-adjusted life years (DALYs) for 291 diseases and injuries in 21 regions, 1990-2010: a systematic analysis for the Global Burden of Disease Study 2010. The Lancet. 2012;380(9859):2197-223.

2. Hawker GA, Stewart L, French MR, Cibere J, Jordan JM, March L, et al. Understanding the pain experience in hip and knee osteoarthritis-an OARSI/OMERACT initiative. Osteoarthritis Cartilage. 2008;16(4):415-22.

3. Hunter DJ, Schofield D, Callander E. The individual and socioeconomic impact of osteoarthritis. Nat Rev Rheumatol. 2014;10(7):437-41.

4. Turkiewicz A, Petersson IF, Bjork J, Hawker G, Dahlberg LE, Lohmander LS, et al. Current and future impact of osteoarthritis on health care: a population-based study with projections to year 2032. Osteoarthritis Cartilage. 2014;22(11):1826-32.

5. Cross M, Smith E, Hoy D, Nolte S, Ackerman I, Fransen M, et al. The global burden of hip and knee osteoarthritis: estimates from the Global Burden of Disease 2010 study. Annals of the Rheumatic Diseases. 2014;73(7):1323. 
6. Caughey GE, Vitry Al, Gilbert AL, Roughead EE. Prevalence of comorbidity of chronic diseases in Australia. BMC Public Health. 2008;8:221.

7. Hall AJ, Stubbs B, Mamas MA, Myint PK, Smith TO. Association between osteoarthritis and cardiovascular disease: Systematic review and meta-analysis. Eur J Prev Cardiol. 2016;23(9):938-46.

8. Veronese N, Cereda E, Maggi S, Luchini C, Solmi M, Smith T, et al. Osteoarthritis and mortality: A prospective cohort study and systematic review with meta-analysis. Seminars in Arthritis and Rheumatism. 2016;46(2):160-7.

9. Kraus VB, Sprow K, Powell KE, Buchner D, Bloodgood B, Piercy K, et al. Effects of Physical Activity in Knee and Hip Osteoarthritis: A Systematic Umbrella Review. Medicine and science in sports and exercise. 2019;51(6):1324-39.

10. Ton J, Perry D, Thomas B, Allan GM, Lindblad AJ, McCormack J, et al. PEER umbrella systematic review of systematic reviews: Management of osteoarthritis in primary care. Can Fam Physician. 2020;66(3):e89-e98.

11. World Health Organization. Global Recommendations on Physical Activity for Health 2011 [cited 2020 May 27]. Available from: https://www.who.int/dietphysicalactivity/publications/recommendations18_64yearsold/en/.

12. Tudor-Locke C, Craig CL, Brown WJ, Clemes SA, De Cocker K, Giles-Corti B, et al. How many steps/day are enough? For adults. Int J Behav Nutr Phys Act. 2011;8:79.

13. Thoma LM, Dunlop D, Song J, Lee J, Tudor-Locke C, Aguiar EJ, et al. Are Older Adults With Symptomatic Knee Osteoarthritis Less Active Than the General Population? Analysis From the Osteoarthritis Initiative and the National Health and Nutrition Examination Survey. Arthritis Care and Research. 2018;70(10):1448-54.

14. Chang AH, Song J, Lee J, Chang RW, Semanik PA, Dunlop DD. Proportion and associated factors of meeting the 2018 Physical Activity Guidelines for Americans in adults with or at risk for knee osteoarthritis. Osteoarthritis Cartilage. 2020.

15. Kim IJ, Kim HA, Seo YI, Jung YO, Song YW, Jeong JY, et al. Prevalence of knee pain and its influence on quality of life and physical function in the Korean elderly population: a community based crosssectional study. J Korean Med Sci. 2011;26(9):1140-6.

16. Rosemann T, Kuehlein T, Laux G, Szecsenyi J. Osteoarthritis of the knee and hip: a comparison of factors associated with physical activity. Clin Rheumatol. 2007;26(11):1811-7.

17. Lee J, Song J, Hootman JM, Semanik PA, Chang RW, Sharma L, et al. Obesity and other modifiable factors for physical inactivity measured by accelerometer in adults with knee osteoarthritis. Arthritis care \& research. 2013;65(1):53-61.

18. Veenhof C, Huisman PA, Barten JA, Takken T, Pisters MF. Factors associated with physical activity in patients with osteoarthritis of the hip or knee: a systematic review. Osteoarthritis Cartilage. 2012;20(1):6-12.

19. Batsis JA, Germain CM, Vásquez E, Zbehlik AJ, Bartels SJ. Physical Activity Predicts Higher Physical Function in Older Adults: The Osteoarthritis Initiative. Journal of Physical Activity and Health. 
2016;13(1):6-16.

20. Wellsandt E, Golightly Y. Exercise in the management of knee and hip osteoarthritis. Curr Opin Rheumatol. 2018;30(2):151-9.

21. Golightly YM, Allen KD, Caine DJ. A comprehensive review of the effectiveness of different exercise programs for patients with osteoarthritis. Phys Sportsmed. 2012;40(4):52-65.

22. Willett M, Duda J, Fenton S, Gautrey C, Greig C, Rushton A. Effectiveness of behaviour change techniques in physiotherapy interventions to promote physical activity adherence in lower limb osteoarthritis patients: A systematic review. PLoS One. 2019;14(7):e0219482.

23. Brickwood K-J, Watson G, O'Brien J, Williams AD. Consumer-Based Wearable Activity Trackers Increase Physical Activity Participation: Systematic Review and Meta-Analysis. JMIR Mhealth Uhealth. 2019;7(4):e11819.

24. Franssen WMA, Franssen GHLM, Spaas J, Solmi F, Eijnde BO. Can consumer wearable activity tracker-based interventions improve physical activity and cardiometabolic health in patients with chronic diseases? A systematic review and meta-analysis of randomised controlled trials. International Journal of Behavioral Nutrition and Physical Activity. 2020;17(1):57.

25. Davergne T, Pallot A, Dechartres A, Fautrel B, Gossec L. Use of Wearable Activity Trackers to Improve Physical Activity Behavior in Patients With Rheumatic and Musculoskeletal Diseases: A Systematic Review and Meta-Analysis. Arthritis care \& research. 2019;71(6):758-67.

26. Shin G, Jarrahi MH, Fei Y, Karami A, Gafinowitz N, Byun A, et al. Wearable activity trackers, accuracy, adoption, acceptance and health impact: A systematic literature review. Journal of Biomedical Informatics. 2019;93:103153.

27. World Health Organization. Adherence to long-term therapies: evidence for action. Section 1 [Internet]. World Health Organization; 2003.

28. Pisters MF, Veenhof C, Schellevis FG, Twisk JWR, Dekker J, De Bakker DH. Exercise adherence improving long-term patient outcome in patients with osteoarthritis of the hip and/or knee. Arthritis care \& research. 2010;62(8):1087-94.

29. Jacquemin C, Servy H, Molto A, Sellam J, Foltz V, Gandjbakhch F, et al. Physical Activity Assessment Using an Activity Tracker in Patients with Rheumatoid Arthritis and Axial Spondyloarthritis: Prospective Observational Study. JMIR Mhealth Uhealth. 2018;6(1):e1.

30. Paxton RJ, Forster JE, Miller MJ, Gerron KL, Stevens-Lapsley JE, Christiansen CL. A Feasibility Study for Improved Physical Activity After Total Knee Arthroplasty. J Aging Phys Act. 2018;26(1):7-13.

31. Dreher N, Hadeler EK, Hartman SJ, Wong EC, Acerbi I, Rugo HS, et al. Fitbit Usage in Patients With Breast Cancer Undergoing Chemotherapy. Clin Breast Cancer. 2019;19(6):443-9.e1.

32. Hartman SJ, Nelson SH, Weiner LS. Patterns of Fitbit Use and Activity Levels Throughout a Physical Activity Intervention: Exploratory Analysis from a Randomized Controlled Trial. JMIR Mhealth Uhealth. 2018;6(2):e29.

33. Skrepnik N, Spitzer A, Altman R, Hoekstra J, Stewart J, Toselli R. Assessing the Impact of a Novel Smartphone Application Compared With Standard Follow-Up on Mobility of Patients With Knee 
Osteoarthritis Following Treatment With Hylan G-F 20: A Randomized Controlled Trial. JMIR Mhealth Uhealth. 2017;5(5):e64.

34. Clinical trials: Active@Work- Optimizing physical activity at work [Internet]. 2017 [cited 2020-08-17]. Available from: https://clinicaltrials.gov/ct2/show/NCT03354091? term=fitbit\&cntry=SE\&draw=2\&rank=1.

35. Thorstensson CA, Garellick G, Rystedt H, Dahlberg LE. Better Management of Patients with Osteoarthritis: Development and Nationwide Implementation of an Evidence-Based Supported Osteoarthritis Self-Management Programme. Musculoskeletal Care. 2015;13(2):67-75.

36. Jönsson T, Eek F, Dell'Isola A, Dahlberg LE, Ekvall Hansson E. The Better Management of Patients with Osteoarthritis Program: Outcomes after evidence-based education and exercise delivered nationwide in Sweden. PLoS One. 2019;14(9):e0222657-e.

37. Finansiell ID-Teknik BID AB. BankID 2020 [cited 2020 July 8th]. Available from: https://www.bankid.com/en/.

38. Wallis JA, Webster KE, Levinger P, Taylor NF. What proportion of people with hip and knee osteoarthritis meet physical activity guidelines? A systematic review and meta-analysis. Osteoarthritis Cartilage. 2013;21(11):1648-59.

39. Fitbit. Fitbit help article 2020 [2020-06-23]. Available from: https://help.fitbit.com/articles/en_US/Help_article/1379.

40. Bumgardner W. Why Your Fitbit Active Minutes Mean More Than Your Steps Verywellfit.com2019 [2020-06-23]. Available from: https://www.verywellfit.com/why-active-minutes-mean-more-thansteps-4155747.

41. Fitbit. Fitbit Web API 2020 [2020-06-23]. Available from: https://dev.fitbit.com/build/reference/webapi/activity/.

42. Straiton N, Alharbi M, Bauman A, Neubeck L, Gullick J, Bhindi R, et al. The validity and reliability of consumer-grade activity trackers in older, community-dwelling adults: A systematic review. Maturitas. 2018;112:85-93.

43. Evenson KR, Goto MM, Furberg RD. Systematic review of the validity and reliability of consumerwearable activity trackers. Int J Behav Nutr Phys Act. 2015;12:159.

44. Alharbi M, Bauman A, Neubeck L, Gallagher R. Validation of Fitbit-Flex as a measure of free-living physical activity in a community-based phase III cardiac rehabilitation population. Eur J Prev Cardiol. 2016;23(14):1476-85.

45. Chu AH, Ng SH, Paknezhad M, Gauterin A, Koh D, Brown MS, et al. Comparison of wrist-worn Fitbit Flex and waist-worn ActiGraph for measuring steps in free-living adults. PLoS One. 2017;12(2):e0172535.

46. Reid RER, Insogna JA, Carver TE, Comptour AM, Bewski NA, Sciortino C, et al. Validity and reliability of Fitbit activity monitors compared to ActiGraph GT3X+ with female adults in a free-living environment. J Sci Med Sport. 2017;20(6):578-82. 
47. Roos EM, Roos HP, Lohmander LS, Ekdahl C, Beynnon BD. Knee Injury and Osteoarthritis Outcome Score (KOOS)-development of a self-administered outcome measure. J Orthop Sports Phys Ther. 1998;28(2):88-96.

48. Nilsdotter AK, Lohmander LS, Klässbo M, Roos EM. Hip disability and osteoarthritis outcome score (HOOS)-validity and responsiveness in total hip replacement. BMC musculoskeletal disorders. 2003;4:10-.

49. Thorborg K, Roos EM, Bartels EM, Petersen J, Hölmich P. Validity, reliability and responsiveness of patient-reported outcome questionnaires when assessing hip and groin disability: a systematic review. British journal of sports medicine. 2010;44(16):1186.

50. Collins NJ, Prinsen CA, Christensen R, Bartels EM, Terwee CB, Roos EM. Knee Injury and Osteoarthritis Outcome Score (KOOS): systematic review and meta-analysis of measurement properties. Osteoarthritis Cartilage. 2016;24(8):1317-29.

51. R Studio Team. RStudio: Integrated Development for R. RStudio, Inc., Boston, MA 2016 [2020-06-01]. Available from: http://www.rstudio.com.

52. Jones DM, Hart HF, Crossley KM, Ackerman IN, Kemp JL. What is the Agreement Between Two Generations of Commercial Accelerometer in a Free-Living Environment for Young to Middle-Aged Adults? Journal for the Measurement of Physical Behaviour. 2018;2(2):49-57.

53. Tudor-Locke C, Barreira TV, Schuna JM, Jr. Comparison of step outputs for waist and wrist accelerometer attachment sites. Medicine and science in sports and exercise. 2015;47(4):839-42.

54. Twiggs J, Salmon L, Kolos E, Bogue E, Miles B, Roe J. Measurement of physical activity in the preand early post-operative period after total knee arthroplasty for Osteoarthritis using a Fitbit Flex device. Medical engineering \& physics. 2018;51:31-40.

55. Collins JE, Yang HY, Trentadue TP, Gong Y, Losina E. Validation of the Fitbit Charge 2 compared to the ActiGraph GT3X+ in older adults with knee osteoarthritis in free-living conditions. PLoS One. 2019;14(1):e0211231.

56. Morcos MW, Teeter MG, Somerville LE, Lanting B. Correlation between hip osteoarthritis and the level of physical activity as measured by wearable technology and patient-reported questionnaires. $\mathrm{J}$ Orthop. 2020;20:236-9.

57. van der Bij AK, Laurant MG, Wensing M. Effectiveness of physical activity interventions for older adults: a review. Am J Prev Med. 2002;22(2):120-33.

58. Patel MS, Asch DA, Volpp KG. Wearable devices as facilitators, not drivers, of health behavior change. JAMA. 2015;313(5):459-60.

59. Hermsen S, Moons J, Kerkhof P, Wiekens C, De Groot M. Determinants for Sustained Use of an Activity Tracker: Observational Study. JMIR Mhealth Uhealth. 2017;5(10):e164.

60. Heiberg KE, Figved W. Physical Functioning and Prediction of Physical Activity After Total Hip Arthroplasty: Five-Year Followup of a Randomized Controlled Trial. Arthritis care \& research. 2016;68(4):454-62. 
61. Akoglu H. User's guide to correlation coefficients. Turkish Journal of Emergency Medicine. 2018;18(3):91-3.

62. Fernandes L, Roos EM, Overgaard S, Villadsen A, Søgaard R. Supervised neuromuscular exercise prior to hip and knee replacement: 12-month clinical effect and cost-utility analysis alongside a randomised controlled trial. BMC musculoskeletal disorders. 2017;18(1):5.

63. Dell'Isola A, Jönsson T, Ranstam J, Dahlberg LE, Ekvall Hansson E. Education, Home Exercise, and Supervised Exercise for People With Hip and Knee Osteoarthritis As Part of a Nationwide Implementation Program: Data From the Better Management of Patients With Osteoarthritis Registry. Arthritis care \& research. 2020;72(2):201-7.

64. Macridis S, Johnston N, Johnson S, Vallance JK. Consumer physical activity tracking device ownership and use among a population-based sample of adults. PLoS One. 2018;13(1):e0189298-e.

65. Whitaker C, Stevelink S, Fear N. The Use of Facebook in Recruiting Participants for Health Research Purposes: A Systematic Review. Journal of medical Internet research. 2017;19(8):e290.

66. Imboden MT, Nelson MB, Kaminsky LA, Montoye AH. Comparison of four Fitbit and Jawbone activity monitors with a research-grade ActiGraph accelerometer for estimating physical activity and energy expenditure. British journal of sports medicine. 2018;52(13):844-50.

\section{Figures}

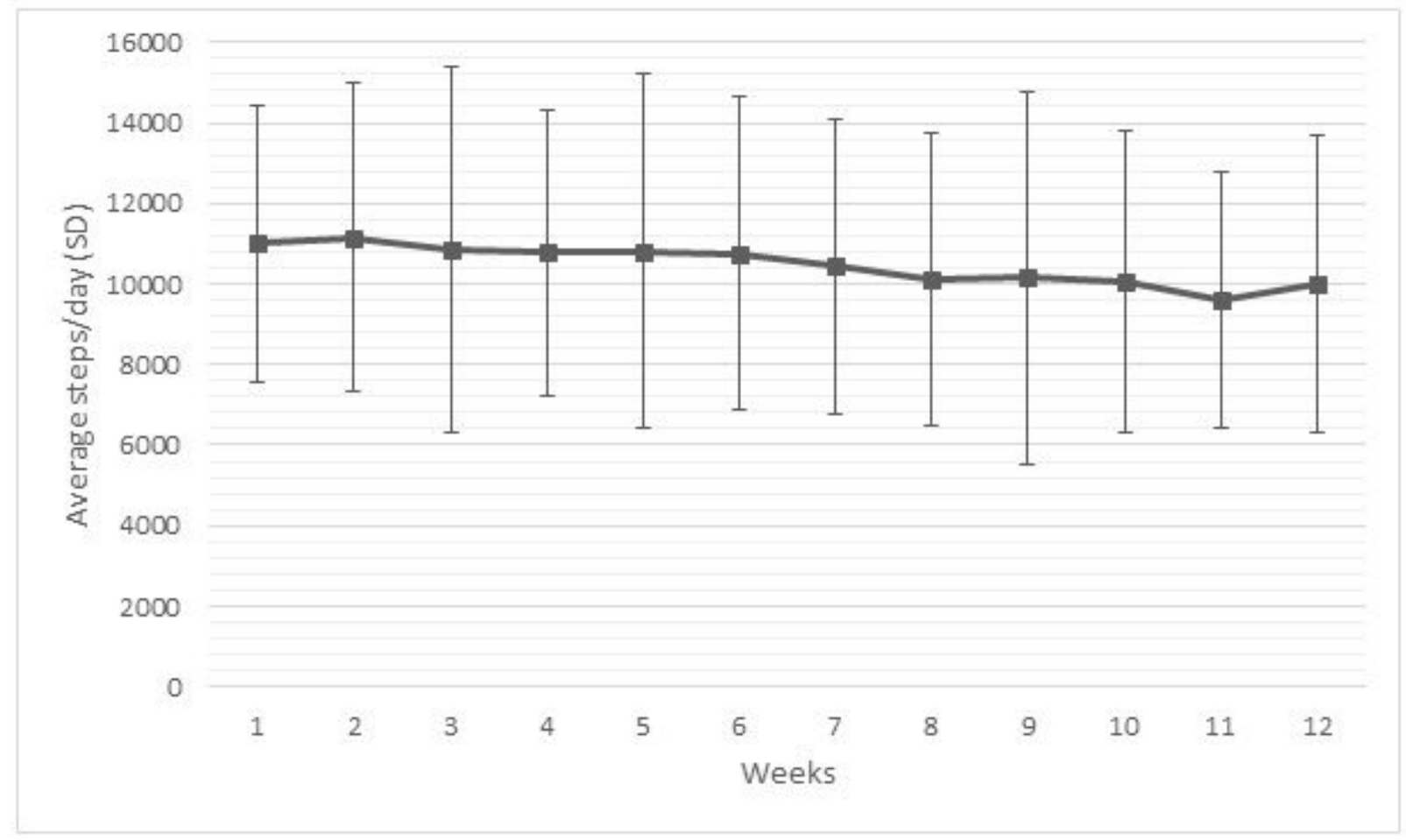

\section{Figure 1}

Mean number of steps per day during the intervention $(n=75)$. 


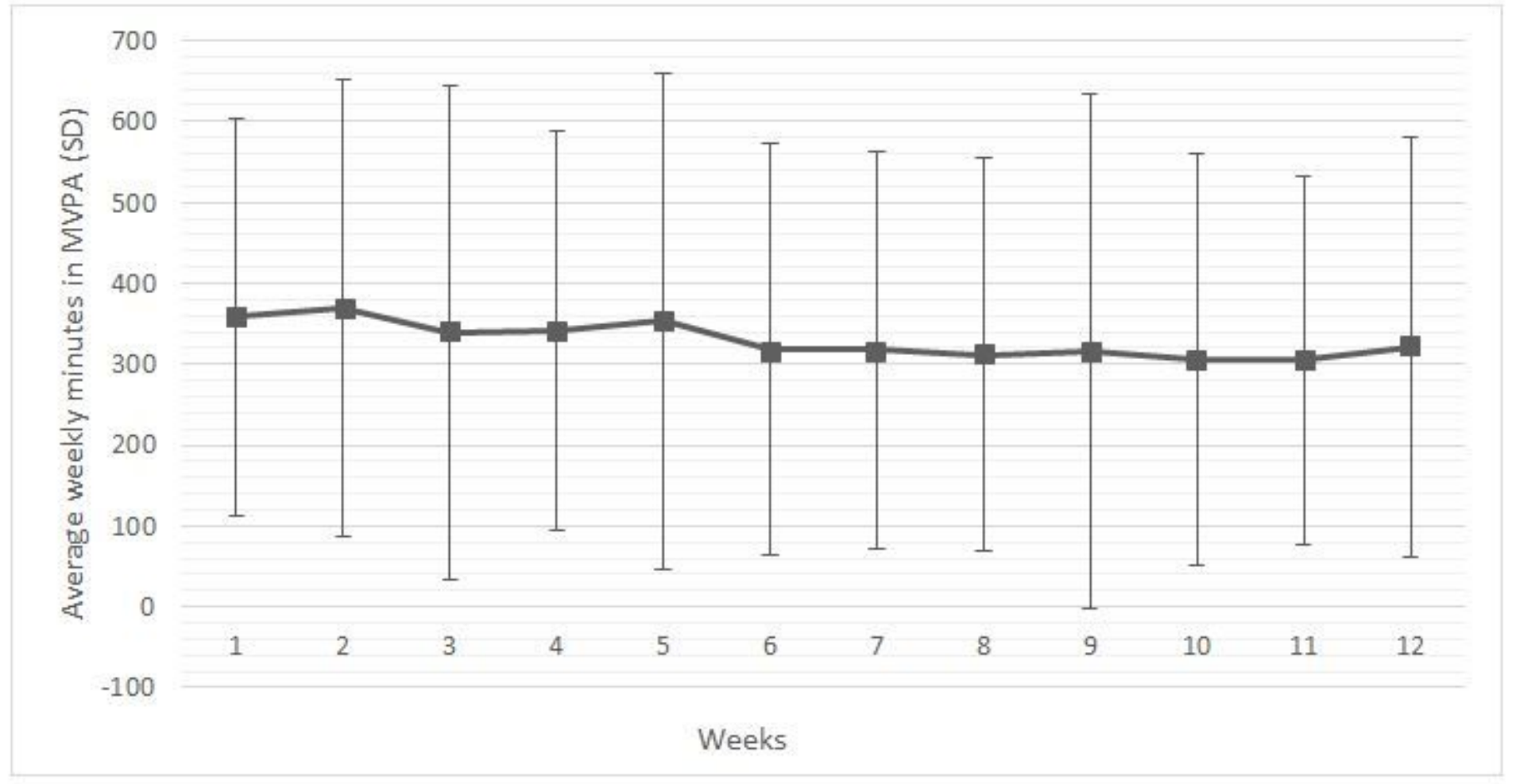

Figure 2

Mean number in MVPA (SD) per week during the intervention $(n=75)$.

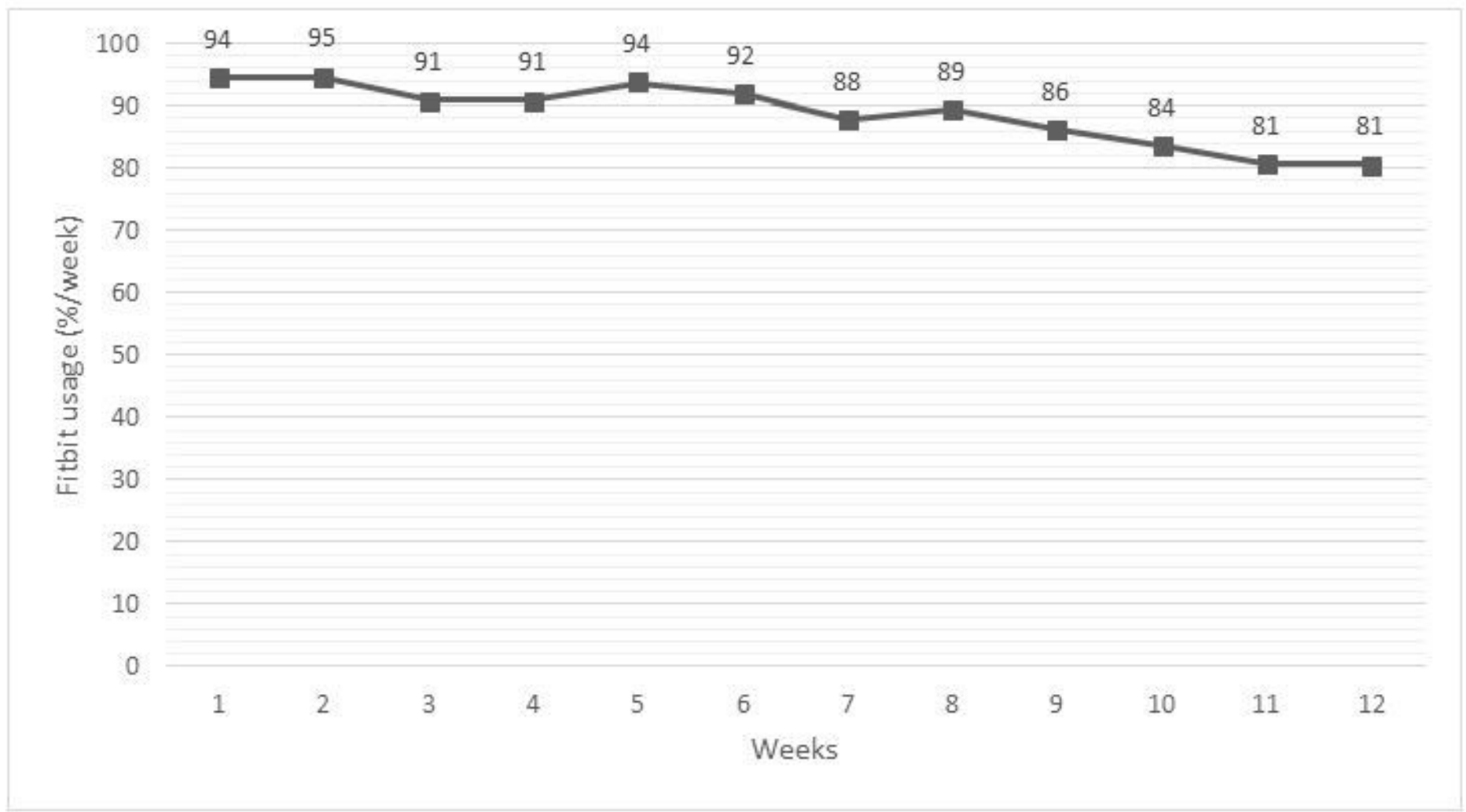

Figure 3 
Fitbit usage on average (percentage per week) during the intervention $(n=75)$ 